\title{
An Adaptive Data Aggregation Algorithm in Wireless Sensor Network with Bursty Source
}

\author{
Kumar PADMANABH, Sunil Kumar VUPPALA \\ Software Engineering and Technology Labs (SET Labs) Infosys Technologies Limited, Bangalore, India \\ E-mail: \{Kumar_padmanabh, sunil_vuppala\}@infosys.com \\ Received April 20, 2009; revised May 25, 2009; accepted June 30, 2009
}

\begin{abstract}
The Wireless Sensor network is distributed event based systems that differ from conventional communication network. Sensor network has severe energy constraints, redundant low data rate, and many-to-one flows. Aggregation is a technique to avoid redundant information to save energy and other resources. There are two types of aggregations. In one of the aggregation many sensor data are embedded into single packet, thus avoiding the unnecessary packet headers, this is called lossless aggregation. In the second case the sensor data goes under statistical process (average, maximum, minimum) and results are communicated to the base station, this is called lossy aggregation, because we cannot recover the original sensor data from the received aggregated packet. The number of sensor data to be aggregated in a single packet is known as degree of aggregation. The main contribution of this paper is to propose an algorithm which is adaptive to choose one of the aggregations based on scenarios and degree of aggregation based on traffic. We are also suggesting a suitable buffer management to offer best Quality of Service. Our initial experiment with NS-2 implementation shows significant energy savings by reducing the number of packets optimally at any given moment of time.
\end{abstract}

Keywords: Data Aggregation, Data Fusion, Congestion Control, Buffer Overflow, End to End Delay

\section{Introduction}

Wireless sensor network (WSN) is a network of sensor nodes. The main constituents of the WSN nodes are the communication devices (i.e. receiver and transmitter), a small Central Processing unit (CPU), a sensing device and a battery. The sensor node senses and gathers information from the surroundings; the CPU executes some control instructions and the communication unit sends the information to the base station through the network of such a large number of nodes.

WSN is distributed in nature and an event based system. Due to size and battery power limitations, these devices typically have limited storage capacity, limited energy resources, and limited network bandwidth. Due to these limitations, WSN differs from traditional communication networks in several ways. These limitations of sensor nodes demand specialized optimization techniques. Typically in WSN applications, a large number of Sensor Nodes (SNs) are covered over the specific target area in close proximity to each other. In such de- ployments, spatial correlation of data is observed where neighboring sensor nodes report data values with a high degree of correlation.

Another kind of correlation observed in sensed environmental data is the temporal correlation of data where the successive sensed parameter values are found to be identical and varies slowly except in the case of unexpected events [1,2].

The spatial and temporal correlations of the WSN data can be exploited favorably for the development of efficient communication protocols in the WSN. Moreover, there is redundancy in the sensor data. The communication cost imposed due to redundant data is unnecessarily consumes lifetime of the nodes and bandwidth. In wireless sensor networks, several information can be combined together and represented by same number of bits. Once this is done the energy consumption in the communication process will be reduced. This process is known as data aggregation. Data aggregation schemes are the most popular way of using the correlation in sen- 
sor data.

Data produced by nodes in the network propagates through other nodes in the network via wireless links. When compared to local processing of data, wireless transmission is extremely expensive. Researchers estimated that sending a single bit over radio is at least three orders of magnitude more expensive than executing a single instruction. With the new developments in the hardware of the motes, increasing memory size is giving us the chance to process the data, perform buffer management operations, so as to reduce the number of transactions over the radio.

For Scalability and flexibility of WSN applications, we need to consider this data aggregation as this results in energy saving and optimized performance. Indeed, several research efforts have been proposed in different forms of aggregation to achieve energy efficiency [1-4].

The aggregation process can be lossless or lossy. In lossless aggregation, more information is embedded into a single packet (instead of one packet for every information) thereby combining all headers into single header and same data bits. In lossy aggregation many data packets are passed through aggregation function that generates a single packet which has no information about the original data. These functions are computed by the intermediate nodes based on the data received. Thus, at each intermediate node, the amount of outgoing data is considerably lower than the amount inputted, resulting in increase of computational overhead thereby decreasing the transmitted data. The degree of aggregation (DoA) is defined as the ratio of number of bits present in all the packets considered for aggregation in one round of aggregation and the number of bits present in the aggregated packet.

There are two different types of routing in WSN literature, namely address centric and data centric. Data centric routing [1] is used as one of the key techniques to support in-network aggregation. Based on the data rather than the data sources and destinations, data centric routing aims to find path from multiple sources to a single destination that promote data aggregation.

Another approach is using hierarchies, where sensor nodes are usually organized into clusters. To perform the data aggregation nodes communicate with each other and form the clusters in order to share their sensed data. Even though such energy savings are desirable, data aggregation is sensitive with delay.

WSNs have wide range of applications. We focus on data aggregation technique that target all classes of sensor network applications from monitoring to industrial grade applications.

The rest of this paper is organized as follows. In Section 2, we give an overview of data aggregation techniques in WSN from the literature and motivation for our work. We present system description and parameters in Section 3 and our approach is discussed in Section 4. Results and graphs are analyzed in Section 5 and finally the paper is concluded in Section 6.

\section{The Related Work, Motivation and Contribution}

Previous studies have proved that substantial energy savings are not only possible but essential for the success of wireless sensor networks [1]. We analyze some previous and on-going research efforts to put our work in perspective. The delay which occurred in the process of aggregation, (termed as aggregation delay) is a function of number of hops between the destination and the farthest source, and depends upon the aggregation parameter such as degree of aggregation, which will be defined in the next section. To maximize the degree of aggregation within the network, data tend to be routed through the paths that promote aggregation, rather than shortest path, which contributes additional delay.

The authors of [1] dealt with the performance issues of sensor data aggregation. They have presented a technique for delay energy trade-off in the presence of non-trivial (time consuming) aggregation. This is a mechanism to perform data centric aggregation. In their algorithm they used application specific knowledge which in turns provides a means to augmenting throughput. One of the limitations is due to its application specific approach. This algorithm is not adaptive.

The authors in [2] proposed an algorithm of aggregation which is a variant of directed diffusion. In this, intermediate nodes collect data for a specific amount of time or till they collect a fixed amount of data and send them for aggregation. The accuracy of aggregation will depend on the delay allowed at the intermediate nodes, which is specified by the application. This can improve path sharing and attain significant energy savings when the network has higher nodal density compared with the opportunistic approach. However, the idea is limited to specific amount of time or specific volume of data which is application dependent.

The authors of [3] investigate the tradeoff in the presence of both data aggregation and topology control (through the sleep/active dynamics of sensor nodes). In these data aggregation technologies, all aggregator nodes would wait for a fixed-period of time before performing aggregation operation. So when the time triggers, the aggregation nodes can receive responses from all of its children. This approach can save more energy consumption, but bring larger latency to the whole network.

The authors in [4] study the energy-accuracy tradeoff under two different types of aggregation: one is snapshot aggregation which is performed once, and other one is periodic aggregation which is regularly performed. The 
authors claim completely distributed and localized (nodes exchange information only with immediate one hop neighbors) algorithm, however the parent should receive an exact number of messages, equal to the number of its children and the final result is only available at the user node. Snapshot aggregation on the other hand is very sensitive to the stability of the hierarchical structure.

The work by the authors in [5] provided a new stochastic decision framework to study the fundamental energy-delay tradeoff in distributed data aggregation. Adaptive real-time dynamic programming (ARTDP) is asynchronous value iteration scheme and is suitable for on-line implementation only. This scheme might be good to have energy-delay trade-off case but Adaptive Application-Independent Data Aggregation (AIDA) [6] offer better energy benefits than this scheme. The authors of paper [6] describe an aggregation scheme that adaptively performs application independent data aggregation in a time sensitive manner. AIDA performs lossless aggregation by concatenating network units into larger payloads that are sent to the MAC layer for transmission. This may not suite all the applications.

Some aggregate functions require the concatenation of all readings to be returned to the host node. For example, in order to accurately determine the median value in a network [7], the host node must know all the values. In this case, it may still be possible to reduce the size and number of messages by applying compression. Researchers propose a unique data structure called a Quantile Digest (q-digest), which provides approximate results that adhere to a strict error bound. But it is a good approximation scheme when there are wide variations in frequencies of different values.

The work by authors of [8] handles the case of lossy aggregation while bounding the number of messages transmitted in the network. They propose a Marginal Gains Adjustment (MGA) algorithm for the problem of bandwidth constrained aggregate continuous queries over sensor network. This does not consider all cases of aggregation and is not adaptive in nature.

Sometimes application specific aggregation will be giving better results rather than the general schemes as it can understand the environment conditions better. So we need to consider some application knowledge and propose a general purpose aggregation scheme.

\subsection{Motivation}

Even though several research works in the literature have discussed the problems and approaches of developing data aggregation processes mainly for energy, bandwidth and memory space savings by minimizing the data transferred in sensor networks [1,2]), however authors of these papers fail to address following practical problems:

Quality of Service (QoS) issues in data aggregation: In sensor network there are several types of data. Namely normal hello packets, normal sensor data packets, some important alert data packets and control messages from the base station. The control message from the base station and the alert sensitive data packets are very important in nature and QoS provided to these packets should be better than others.

Adaptive mechanism: The parameter of the data aggregation such as DoA, QoS cannot be decided and fixed due to the burst nature of the sensor network. It should be adaptable enough. Feedback should be there to make the system controlled and adaptable. Though there is some paper available but they don't address QoS and adaptable aggregation simultaneously.

Scheduling: In the process of addressing QoS, we need to schedule the packets and apply some of the buffer management policies before applying aggregation process.

Most of the proposals in the literature give modeling and simulation of the WSN scenario for various parameters like energy, priority, delay, degree of aggregation supported with the mathematical proofs. The authors of these papers have considered either distinct parameter in each piece of work separately or they have considered only few parameters together $[1,2]$.

Moreover these proposed methods are too complex to be implemented in hardware of current state of the art. Although several schemes for programming and data aggregation in WSNs have been proposed in literature, few actually provide experimental validation and performance evaluation $[5,6]$.

So there is a need to design a data aggregation mechanism in WSN by considering different QoS parameters and take the feedback mechanism to make the system adaptive and save the energy. This general purpose data aggregation should be able to apply for all WSN applications, considering the priority information and application knowledge for aggregation function.

Our approach is to have buffer management in the aggregator nodes to make the adaptive algorithm obeys the rule that degree of aggregation is proportional to number of packets. Special packet formats are considered in the aggregation. So this approach can be used for wide range of sensor applications.

\subsection{Contribution of This Paper}

There are considerable amount of work in data aggregation available in existing literature. Authors of these papers dealt with application dependent or adaptable technique, QoS issues, related techniques in separately. In this scenario following is the contribution of this paper:

1) Lossy Lossless Aggregation: In the same algorithm lossy and lossless aggregation has been taken care. Depending upon the requirement algorithm switched from 
lossy to lossless.

2) Controlled Degree of Aggregation: The degree of aggregation is control parameter and existing number of packets in the buffer determine the instantaneous value of degree of aggregation.

3) Buffer Management: In the same algorithm we have taken care of buffer management which optimizes the QoS by minimizing the packet loss due to buffer overflow. this.

The above three points are our unique contribution in

\section{System Desctipiton}

We consider two types of nodes in our system, normal nodes and aggregating nodes. Normal nodes do not perform aggregation. They sense the data and send it to the sink. They also forward the data generated by other nodes. Aggregating nodes work as normal nodes and perform aggregation. Only local aggregation can be done at normal nodes.

An aggregator receives the data from one or more normal nodes, performs an aggregation based on the algorithm and then forwards the aggregated packet. In WSN, data from all of the nodes are supposed to be shipped to the base station only. Thus a base station in the WSN is a typical sink where the data reaches finally. Actually this base station connects the individual sensor node to outside world.

In our system we consider following four types of packets:

Hello packets which consists of the information about the source nodes and may contain the routing information. It does not contain any sensor data or any other data.

The control packets contain some of the control parameters. It may originate from the base station or from other nodes. The control parameter may be some system control instruction or to set some flag or otherwise.

Normal data packets: In the sensor network the data packets are formed with sensor reading and headers. Regular messages are those messages that contain such sensor data which fall in the expected range. Typically it is the instantaneous sensor reading. In this case sensing is being done as a regular practice which occurs without any event of interest.

Critical data packets: Critical data packets are those packets which contains sensor data and header. This sensor data is generated with an event of interest. For example, the normal temperature of office workplace is $25^{\circ} \mathrm{C}$. A packet with sensor reading of $25^{\circ} \mathrm{C}$ will be known as normal packet. However if the sensor reads a temperature of $75^{\circ} \mathrm{C}$ it will be an event of interest and the packet which contains this reading will be termed as critical packet.
We assume whether a particular node will work as a normal node or aggregator nodes is decided by some technique which is not in the scope of this work.

Typically there are two way of aggregation. Firstly extracting the sensor data from the packet and considering many such sensor data to pass through an aggregation function to get a single data. For example if the sensor data is temperature reading then we can consider many temperature readings to take average of them. Thus, before aggregation we have multiple sensor data however after aggregation we have a single average value. When this average value is used to form a packet we call it as an aggregated packet. This aggregated packet is lossy. Because at receiving end we cannot reproduce the original sensor data with the average value of reading. Therefore it is called as lossy aggregation. However there is another technique in which sensor readings are extracted from multiple packets and they are put into single packet with one header only. Here nothing is lost however we are getting rid of header information. The packet length will be variable in this case. This is lossless aggregation.

In our system we consider both lossy and lossless types of aggregation. To choose between lossy and lossless is completely application dependent. However general rule is that when packet size is not fixed, we can go for lossless aggregation and when we have an optimally designed fixed size packet we can go for lossy aggregation.

In our system we have considered two different level of aggregation taking place at different nodes. It starts from the source node itself. The first among these two levels of aggregation is local aggregation. Here any particular node generates data from sensor readings and put them into a packet. Nodes may decide to put more than one sensor data in single packet; they may take average, min-max of some of the data actually depending upon the application and then put them into a single packet. So the number of data packets is reduced and information of many possible data packets is embedded into a single data packet. We call it as local aggregation or level-1 aggregation. It is to be noted that though it is a local aggregation, this is a global policy of data aggregation. It means all other nodes of similar kind will do same aggregation throughout the network.

Second level of aggregation happens with the data packets of locally aggregated data down the line towards the base station. Thus it may happen at any intermediate node from the source node to the base station. In this second level of aggregation some aggregation function is applied to the data streaming from various source nodes to these level-2 aggregator nodes for lossy aggregation or sensor data are extracted from the packets to put them into single packet for lossless aggregation. This again depends upon the application. In this case aggregation 
may be done on one kind of sensor data.

\subsection{Useful Parameters Considered in the System}

The performance evaluation of the data aggregation mechanism can be done by analyzing some of the parameters. In our system we consider following parameters:

\subsubsection{Degree of Aggregation}

We define degree of aggregation as a ratio of total number of number of bits in all packets considered for one round of aggregation process and total number of bits in aggregated packets.

Let us consider that $X$ is the number of data bits in the packet and $H$ is the number of header bits in a single packet. Thus if $n$ number of packets are considered for aggregation in one round of algorithm of aggregation, let us consider the lossy and lossless aggregation case separately to define degree of aggregation formally,

Lossy aggregation: In this case $n$ numbers of sensor data are passed through aggregation function to get a single packet. Additionally $z_{1}$ number of additional bits will be added to form the aggregated bits. $z_{1}$ is the number of bits required to carry the aggregation information like average value, statistical value, number of packets involved in the aggregation. This $z_{1}$ can be fixed for a particular application. These $z_{1}$ bits are used to decode the aggregated sensor data at the sink. Therefore DoA in this case will be defined as: $D o A=\frac{n(X+H)}{X+H+z_{1}}$, for a very minimal additional bits as an identifier i.e. $z_{1} \square n(X+H)$. The degree of aggregation will be reduced to $n$ itself.

In the case of lossless data aggregation the degree of aggregation is defined as similarly. However the number of bits after aggregation will be reduced to $n X+H+z_{2}$. Therefore degree of aggregation for lossless aggregation can be defined as $D o A=\frac{n(X+H)}{n X+H+z_{2}}$ it is to be noted that degree of aggregation for lossless case is lesser than its lossy counterpart.

\subsubsection{QoS}

We have considered priority based service to four types of packets defined earlier. We consider hello packet and normal data packets as general packets. Other packets, namely, control packets and critical packets are important packets. The important packets will have priority over the normal packets for service. We apply a special buffer management policy with data aggregation to achieve this. Typically we don't want to have lossy ag- gregation or loose any packets from these high priority packets.

\subsubsection{Packet Format}

To achieve the QoS discussed earlier we have proposed a general packet format which is applicable for both lossy as well as lossless aggregation. In WSN, there is no fixed format for the packet in practice. We are proposing both fixed and variable length packet format.

\subsubsection{Fixed Packet Format}

For lossy aggregation following is the packet format considered. We have a typical OS based header packet type and data field. It is to be noted that data packet have fixed length in this case.

For example, TinyOS [9] default payload is of 29 bytes. TinyOS Header field consists of destination address, type, group id and message length. Rest of the payload is defaulted to 29 bytes. In our packet structure of multi hop routing, along with standard TinyOS header, we have few more fields as additional header, namely source node address, parent node address, hop count, sequence number and last forwarder id. Rest of the payload consists of different sensor analog to digital converter $(\mathrm{ADC})$ values indicating sensor data readings. The packet is represented in associated Figure 1.

As the payload is taken as fixed size for the aggregated packet in lossy aggregation, one extra type field is enough to differentiate normal packet and aggregated packet.

\subsubsection{Variable Length Packet Format}

We propose special adaptable packet format here. The header field will be the same except there will be additional fields in header which will carry information about the length of the packet. The length of data field will be variable so the total length of the packet will be variable in nature and adapt to the current scenario. This is mainly

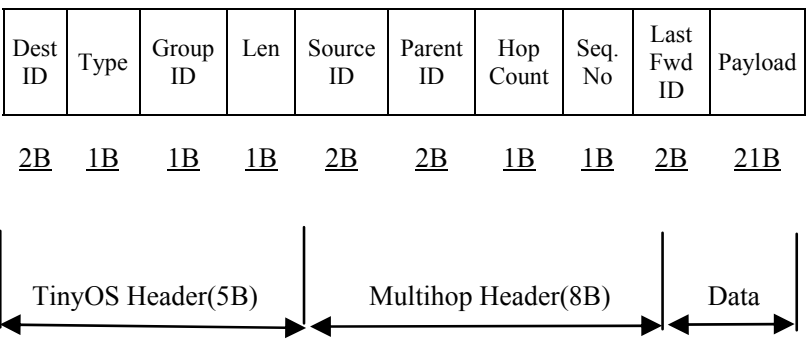

Figure 1. Packet structure for TinyOS with multi hop routing.

\begin{tabular}{|l|l|l|}
\hline Header & Agg. Type & Payload(variable length) \\
\hline
\end{tabular}

Figure 2. Adaptive aggregated packet. 


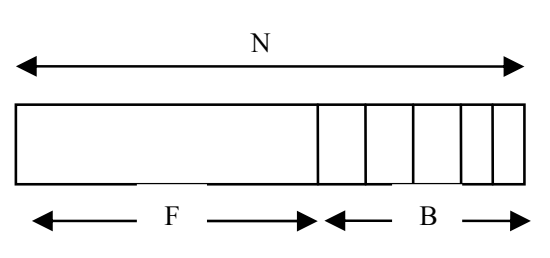

Figure 3. Buffer and DoA relationship.

used for lossless aggregation. However it is to be noted that there is a maximum limit to the length of this payload. For example, in case of TinyOS, the message length can be up to 116 bytes. So there will be different combinations possible to prepare the variable aggregated packet as sensor readings from different nodes need to be sent in a single packet. We shall consider both the type field in the header and the aggregation type in payload to handle different combinations (Figure 2). This variable packet needs to be interpreted correctly at the base station by considering the aggregator type filed. By this way, system can generate aggregated packet on the fly based on the inputs given to the system.

\subsubsection{Special Buffer Management for Data Aggregation} Data aggregation involves combining several sensor readings in intermediate nodes. This in turn requires storing the packets from different sensor nodes and processes them in the memory space available in nodes and outputting aggregated packet. To input the packets from different sensor nodes, we consider buffer space in the aggregator node and to process them we need a special management policy $[10,11]$ so that it can provide specific number of packets to aggregation process after considering type of packet and DoA type.

Buffer acts as a storage for the packets and works similar to a queue. In our system, we consider a temporary buffer and multiple queue system in main buffer. First the input packet reaches the temporary buffer and then caters to different priority queues. We define different queues for different priority packets. For the first queue in the buffer, we push normal and Type-1 critical packets for which aggregation is needed. Second queue is for important packets and third queue is for critical packets. Let us consider that $\mathrm{N}$ is the total space in buffer and $\mathrm{B}$ is the number of packets in the buffer (Figure 3 ). Thus, $\mathrm{F}$ is considered as the difference between $\mathrm{N}$ and $\mathrm{B}$ (i.e. N-B) indicating free space in the buffer [10].

The policy considered in the buffer management follows these rules.

1) General packet processing is on the first come first serve basis.

2) From temporary buffer, the packet is pushed to relevant queue in the main buffer based on the type of packet.

3) A packet is never dropped as long as there is room in the main buffer.

4) A packet from temporary buffer is discarded only if the main buffer is full.

5) DoA is proportional to the number of packets (DOA $\alpha \mathrm{B})$ as shown in the Figure 3.

6) If there is no space for incoming packet, packet of the low priority is dropped from the temporary buffer.

\section{Our Approach}

In this section, we present our approach of adaptive data aggregation based on different parameters mentioned earlier in the paper. In our system, aggregation is performed in two levels after storing and processing the sensor data packets in the buffer.

Hello packets and control packets are processed without aggregation. Aggregation is performed for the normal packets. Based on the application demand, critical data packets can also be aggregated. If the node can take necessary action in response to the event of interest, we may send the critical data packet after the aggregation, referred as Type- 1 critical packets. This is implemented by incorporating necessary functionality inside the node. For these Type-1 critical packets, a control packet is also generated from the node. This control packet could be sending an alarm signal or sending an alert to the corresponding person. For Type- 2 critical packets, no aggregation takes place as the critical data packet is sent to the sink as soon as possible. In this case, the sink responds to the received critical data packet, which is generated for the event of interest from the node.

The sensors sense the data at frequent intervals of time and check for the possibility of any local aggregation before generating the packets. This is referred to as local aggregation. After local aggregation, the packet is generated and enters into the buffer of the next node towards the sink from the input queue. Based on the aggregation mechanism and type of packet, few packets are processed and an aggregated packet is outputted as described in the algorithm. The effectiveness of data aggregation is improved by taking feedback from the system. This feedback contains the number of packets to be considered for aggregation in each round. We consider the feedback and degree of aggregation type in the buffer management to make adaptive aggregation as shown in Figure 4.

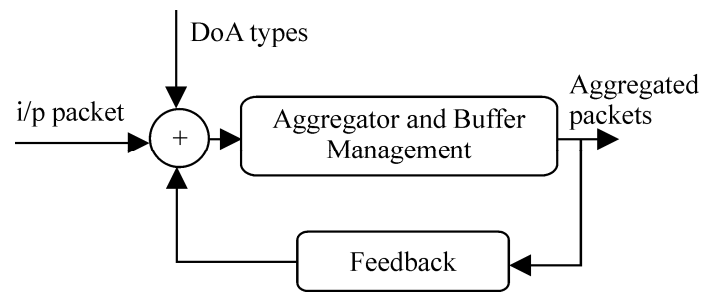

Figure 4. Feedback mechanism in the data aggregation. 


\begin{tabular}{|c|c|}
\hline Range of packets & DoA Type \\
\hline $\mathrm{B}<\mathrm{M} 1$ & 1 \\
\hline $\mathrm{M} 1<\mathrm{B}<\mathrm{M} 2$ & 2 \\
\hline $\mathrm{M} 2<\mathrm{B}<\mathrm{M} 3$ & 3 \\
\hline $\mathrm{M} 3<\mathrm{B}<\mathrm{N}$ & 4 \\
\hline
\end{tabular}

Figure 5. Degree of aggregation type based on count/time and number of packets.

If aggregation is being done in a control environment, then degree of aggregation will not be a fixed parameter in the system. It will be adaptive to the instantaneous requirement of application. Moreover, the system can not aggregate all the packets present in the buffer due to the processing involved, which is delay sensitive. So this leads to requirement of different DoA types. The system can be either packet-count based or time based. The system waits until the buffer reaches the specified number of packets in the count based type, where as in the time based type the system waits for a particular amount of time which in turn decides automatically the value of DoA types from Figure 5.

We choose the number of packets to be aggregated at each instance and are given to the system as feedback so that corresponding DoA type is chosen to decide the aggregation mechanism to be performed. For example, let M1, M2, M3 represent different numbers, which are selected based on the application. If the present number of packets in the buffer is less than M1 choose DoA Type-1. DoA Type-2 is chosen if the number of packets lies between M1 and M2 as shown in Figure 5.

The DoA type and the range of packets can be adaptive based on the feedback from the system so that we can optimize the aggregation output.

For each round of operation, specified number of packets are aggregated based on the above mentioned considerations as described in the algorithm. The resulting aggregated packet is sent as output from the system. This holds good for both lossy and lossless aggregation. From the aggregated packet, we calculate the DoA based on the number of packets involved in the aggregation and type of aggregation like lossy or lossless which is explained in the previous section.

\subsection{The Algorithm}

By considering all the parameters and features mentioned in the last section, we propose an adaptive algorithm for data aggregation in two levels for both lossy and lossless types of aggregation. Level-1 aggregation is being performed locally just after reading the sensor data. However, level-2 aggregation is being performed on the sensor data coming from various nodes. We follow the algo-

rithm for level-1 and level-2 aggregation as explained in Table 1. In level-2 aggregation, we logically divide the system into two phases namely, collection and aggregation phases. Collection phase collects the data to be aggregated where as aggregation phase processes the actual aggregation. The collection and aggregation phase repeat until the system is running. Few steps will be common for both lossy and lossless aggregation.

Let us first consider the level-1 aggregation. The sensor nodes sense the data from the environment at frequent intervals of time. After sensing the data, it checks

Table 1. Algorithm for data aggregation.

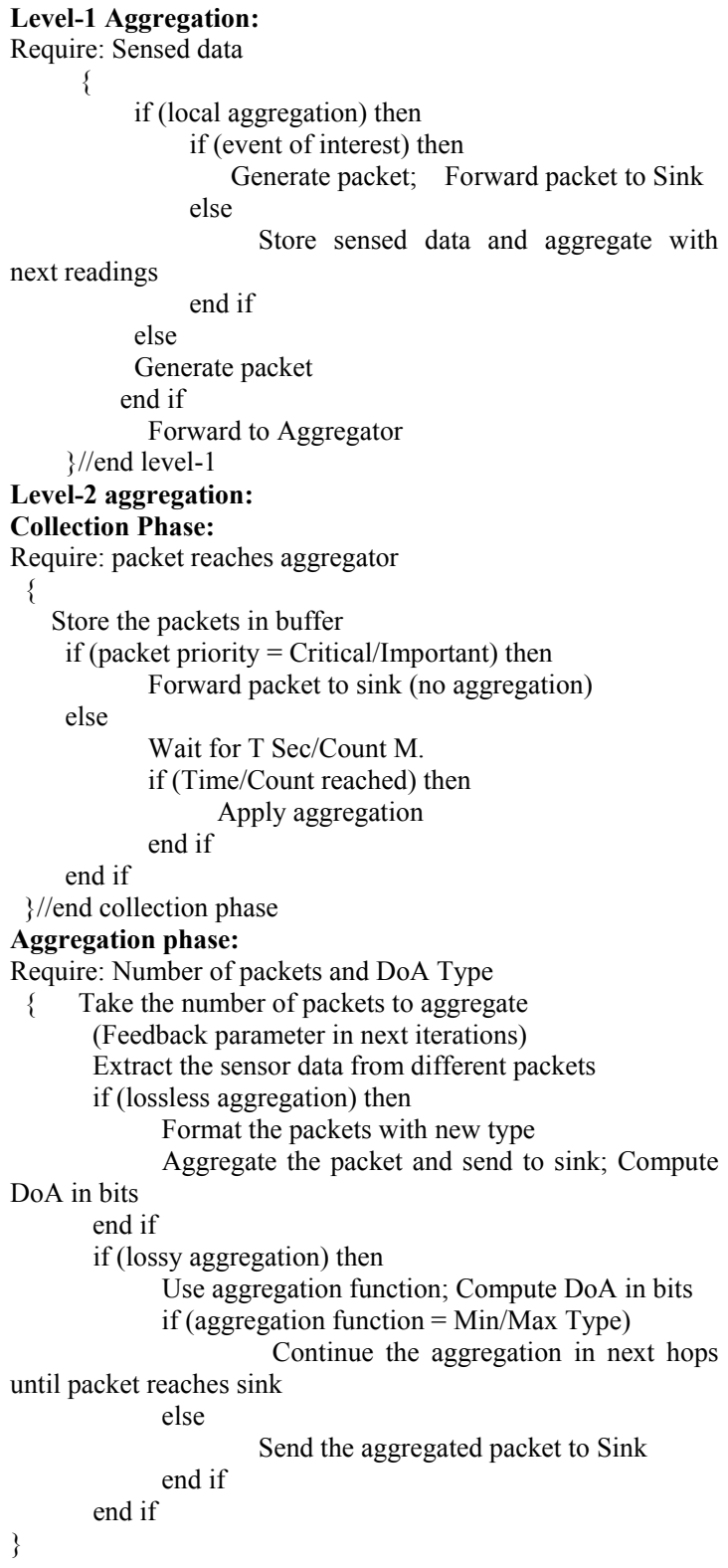


for the need of any local aggregation (level-1) within the node. If local aggregation is possible, it stores the sensor data and waits for the next sensor readings before generating the packet. In case of any event of interest, the packet is generated without waiting for local aggregation and forwarded to the next node towards sink. Otherwise, this node generates the packet and forwards it to the aggregator node. If local aggregation is performed, it is indicated by the type field in the packet format. So at this point, several packets from different nodes reach the aggregator nodes. This is referred as level-1 aggregation.

In the collection phase of level-2 aggregation, the aggregator node collects the packets in the buffer. It checks for the priority of the packets and append the packet in the buffer as per the priority described in earlier section. If the packet is found to be critical or important, they are forwarded from the aggregator towards sink with out any aggregation. In other words those packets are not aggregated at all. In the system, it needs to identify either to follow count based or time based mechanism for the aggregation. In the count based, the system will wait for specific number of incoming packets to be inserted into the buffer. Then it aggregates the fixed number of packets (as per current DoA) from the head of the queue. The choice of count based or time based depends on the application.

Actually in phase-2, DoA type and number of packets to be aggregated are taken as inputs. DoA type is taken from predefined readings of the system as given in Figure 5. The number of packets to be aggregated at any particular moment of time is determined by current space in buffer which is taken as a feedback parameter as shown in Figure 4. All these steps are similar for lossless and lossy both. However, from this point onwards, lossy and lossless aggregation methods differ and are described as follows:

In lossy aggregation, particular number of packets in buffer is considered for aggregation from the collection phase and the sensor readings are extracted from different packets. Then according to requirement of application, a particular aggregation function is selected.

Basically there are two types of aggregation functions possible. Functions like average, standard deviation are limited to one hop only in aggregation process. That means, once the packets are aggregated with this function, no further aggregation is suggested till it reaches the sink. In the other case, functions like minimum, maximum can continue aggregation till it reaches to sink, further reducing the number of packets transferred in the system.

It is to be noted that except the type field for indication of aggregated packet, the size of the packet remains same in this case. At each aggregation step, the DoA is computed in terms of bits. In our system we have considered this for energy saving calculation and to analyze the system performance.
In case of lossless aggregation, the sensor readings are extracted from the packets taken from collection phase. All these sensor readings are aggregated and formatted into a packet with a new type and variable length. The type and length fields describe the packet format to retrieve the readings at the sink. The DoA is computed in terms of bits, at each aggregation step.

Once the packets with or without aggregation reach the sink, it extracts the readings based on the packet type and is used for the application. These steps are described in the algorithm shown in Figure 6 and Table 1.

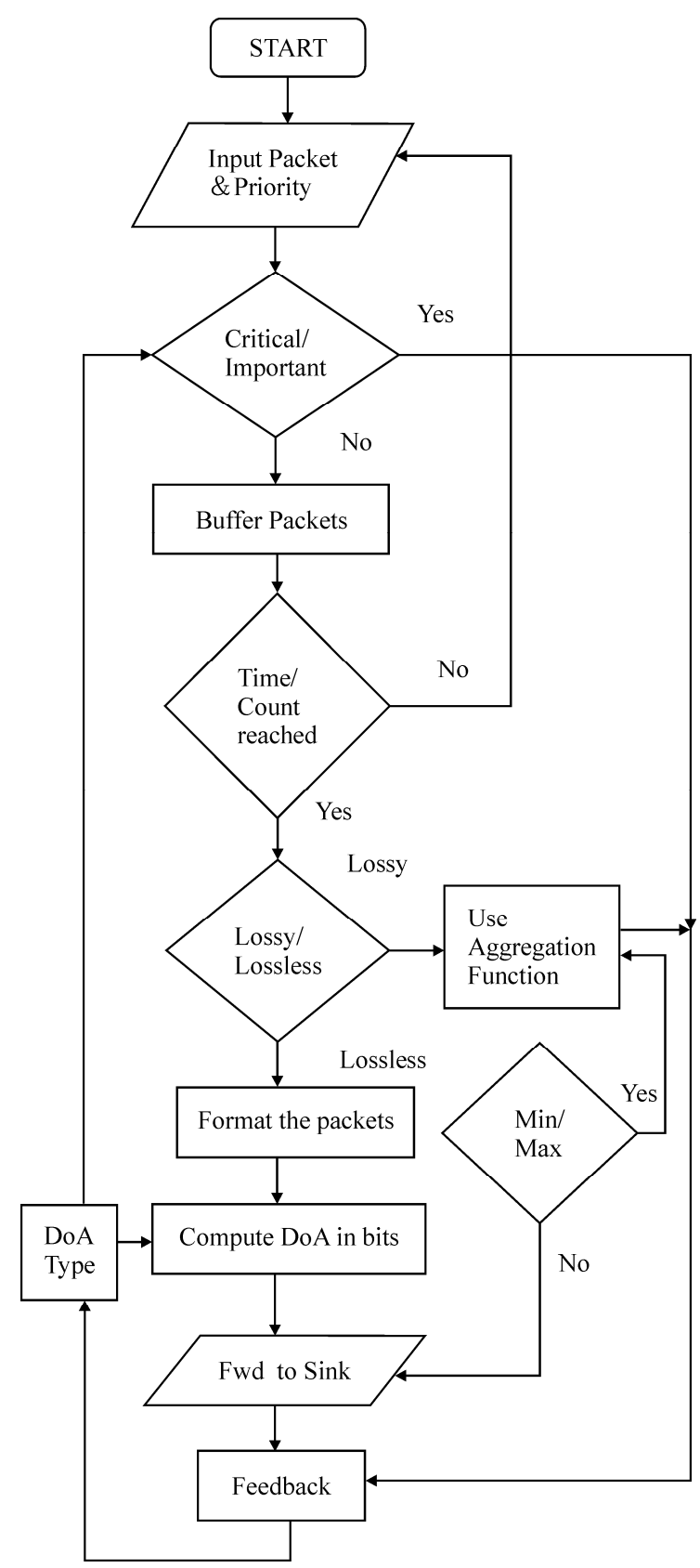

Figure 6. Flow chart of level-2 aggregation. 
Different applications can be taken up to illustrate our scheme. Let us consider temperature monitoring application with average value as the aggregation function as one illustrative application. This is a typical monitoring application using sensor nodes. Nodes are deployed in a room or in an open space where there is a need for monitoring. The sink or base station is located either in the same room where the nodes are deployed or in another room. The packets carrying the sensor data reach the base station in regular intervals of time. The base station process the data and business logic is applied. So the critical data packets should be sent to the base station as soon as possible without the aggregation. Required alerts are raised to the concerned person if the temperature readings are out of bound. Out of bound temperature readings are considered as the event of interest. As the aggregation function considered is average, the aggregation mechanism is considered up to 1 hop level only. After the packets are aggregated, they are sent to sink without further aggregation in the next levels.

In this application, the packet is generated every one second at each node indicating the temperature reading. If there is not much change in the sensor reading from the previous value (up to a reference) we can do local aggregation. After that the packets are generated and reach the aggregator.

Based on the algorithm of lossy aggregation, packets are aggregated and the aggregated packet is indicated as a special type of packet. We follow the table (Figure 7) to choose the DoA type.

Here it is a time based function. For every 10 seconds the aggregation algorithm is called to check the number of packets (B) and DoA type to apply the aggregation mechanism in the system.

\section{Analysis \& Results}

In this section we analyze the results from simulation of our model. Different parameters considered in the system are defined as follows:

Average Delay: Delay is taken as the time each packet is in the buffer in the process of aggregation. Average delay is calculated taking average time each packet spends in the buffer.

Degree of Aggregation: The DoA is defined as the ratio of number of bits present in all the packets considered for aggregation in one round of aggregation and the number of bits present in aggregated packet.

Packet Loss: It indicates the number of packet drops or loss due to buffering of the packets to aggregate in the process of aggregation. Critical packets, important packets and normal packets are treated differently in the buffer and corresponding loss rate is considered.

\begin{tabular}{|c|c|c|}
\hline Range of packets & Time & DoA Type \\
\hline $\mathrm{B}<10$ & $10 \mathrm{sec}$ & 1 \\
\hline $10<\mathrm{B}<20$ & $10 \mathrm{sec}$ & 2 \\
\hline $20<\mathrm{B}<30$ & $10 \mathrm{sec}$ & 3 \\
\hline $30<\mathrm{B}<100$ & $10 \mathrm{sec}$ & 4 \\
\hline
\end{tabular}

Figure 7. Example of time based DoA.

These parameters are considered for different Constant Bit Rate traffic (CBR) traffic, network sizes in both lossy, lossless aggregation based on count and time. We have conducted simulations in Network Simulator (NS-2) [12] to test the performance of our model in large scale. Packet size is taken as 32 bytes, as described in earlier section. Lossless aggregated packet size is variable and maximum size is considered as 116 bytes. So each lossless aggregated packet can accommodate maximum of 20 packets considering 3 sensor readings per each packet. CBR varies from 1 packet/sec to 40 packets/sec. Different network sizes from 10 sensor nodes to 500 sensor nodes are considered in the simulation. Buffer can accommodate a total of 300 packets. For count based, 10 packets are aggregated at a time. In time based, we have taken interval of 1 minute, so the number of packets differs as traffic and network size increases. This buffer is divided into 3 equal parts (100 packets) for critical, important and normal packets. But this memory is sharable among these packets giving the order of preferences, as described in the system description of the paper.

\subsection{Degree of Aggregation for Different Network Sizes}

Here DoA is considered for different network sizes as shown in Figure 8 keeping the CBR as constant and tested for all four possible combinations of lossless count based, lossless time based, lossy count based and lossy

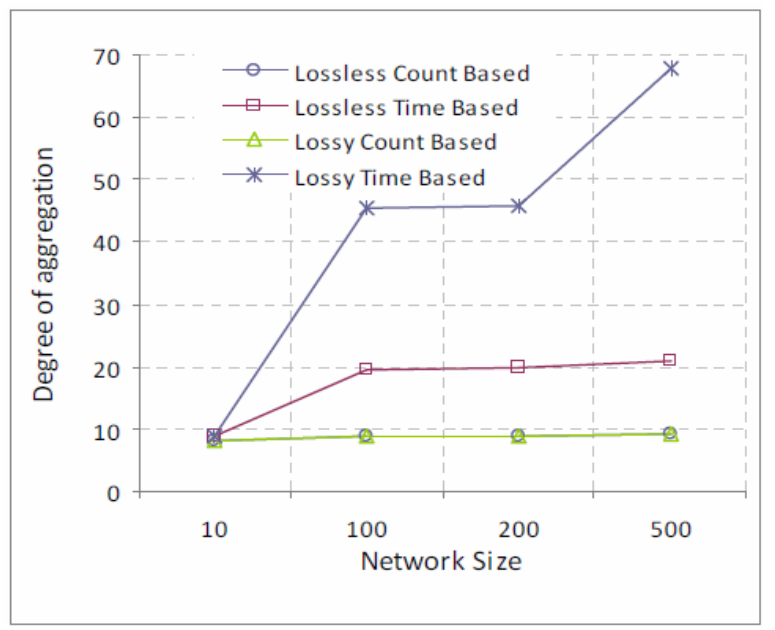

Figure 8. Degree of aggregation for different network sizes. 


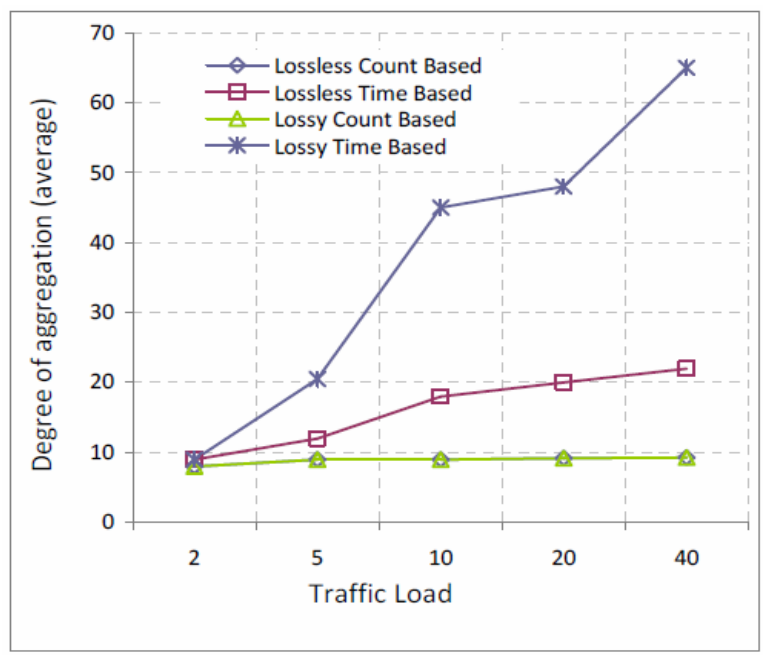

Figure 9. Degree of aggregation for different traffic.

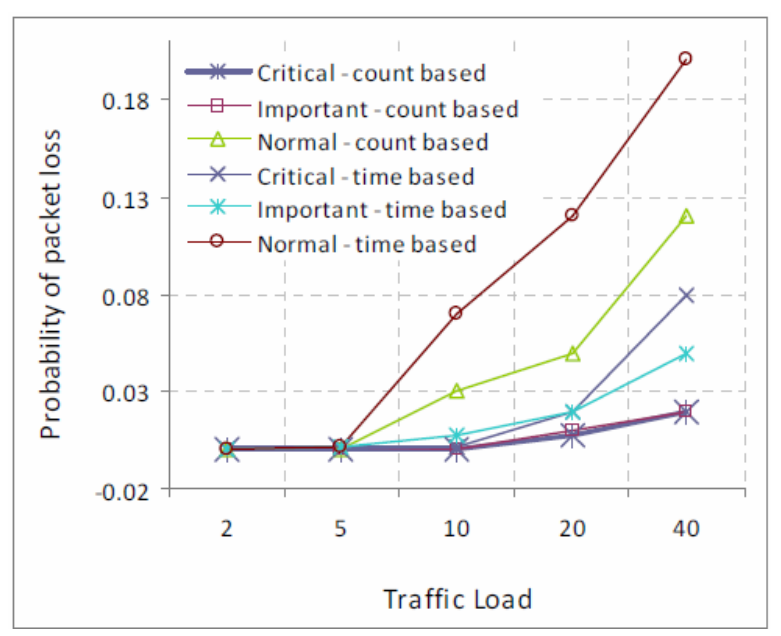

Figure 10. Packet loss for different traffic.

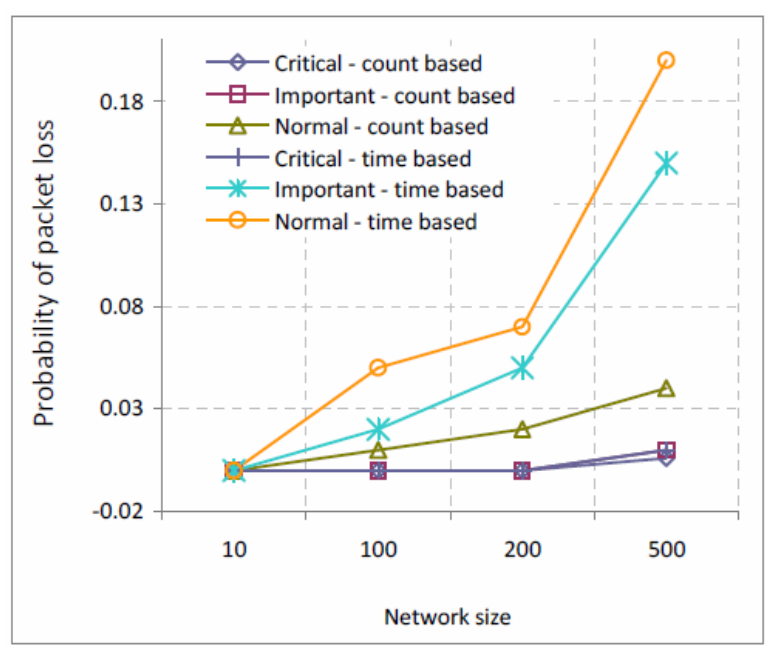

Figure 11. Packet loss for different network sizes. time based aggregations. In count based aggregation, the DoA is in the range of 10 only both for lossy and lossless due to the fact that as soon as count reaches for 10 packets, aggregation is applied. In case of time based aggregation, DoA increases as network size increases based on the table (Figure 5) for different DoA types. In case of lossless aggregation, there is a limit of DoA as it can accommodate maximum of 20 packets, so limiting the DoA around 20. In the case of lossy aggregation, the DoA grows as per the DoA type (Figure 5) and is limited by the buffer size only. It is very evident that our proposed algorithm makes the system adaptable to instantaneous condition and the required aggregated packet is generated with proper DoA.

\subsection{Degree of Aggregation for Different Traffic}

Here DoA is considered for different traffic rates by varying CBR flow as shown in Figure 9 keeping the network size as constant of 100 nodes and tested for all four possible combinations. In count based aggregation, the DoA is in the range of 10 only both for lossy and lossless due to the fact that count of 10 is the limit to trigger the aggregation process. But DoA increases as traffic rate increases in case of time based aggregation. In time based lossless aggregation, DoA is around 20 as described in the first graph. For lossy aggregation, DoA increases as traffic load increases and grows as per the DoA type. In this case, more packets are available for aggregation with increase in traffic load. Only limitation for DoA is the buffer size. Feedback is used at each stage to choose the specific DoA type as mentioned in the algorithm.

\subsection{Packet Loss for Different Traffic and Network Sizes}

In our system, we have different types of packets (critical, important and normal) which are treated differently inside the buffer in the process of aggregation. Our goal is to minimize the loss of packets in the buffer and to have less delay, for which a trade off is required. In Figure 10, packet loss is shown for different traffic load keeping the network size as constant at 100 nodes. In Figure 11, packet loss is shown for different network sizes by keeping the CBR as constant of 10 packets per sec. In both the cases, loss of critical packets is very less initially but as network grows there are a bit of drop in the critical packets. In case of the important packets and normal packets also as traffic rate/network size increases, there is a drop in the packets due to the limitation of buffer size. But packet loss is more in time based when compared to count based as the packets in the buffer are limited in case of count based aggregation mechanism. In count based, aggregation process is triggered by reaching 
a specific number of packets in buffer even more packets are generated in the system. In case of time based more packets reach buffer as traffic load increases, so the loss of packets. Overall, critical packets loss is very minimal, as desired.

\subsection{Average Delay for Different Network Sizes}

Aggregation is applied more frequently in count based therefore least delay is observed. In time based aggregation, more number of packets gets accumulated which is resulted in more delay. However in lossy aggregation, since the number of bits reduced drastically therefore, least delay is there. In lossless aggregation no bits of information is lost therefore more queuing delay is introduced. This is why time based lossless aggregation has highest delay. All four cases can be easily interpreted from Figure 12.

We need a tradeoff between the delay and the DoA keeping the track of packet loss. Even though count based aggregation is having less delay and less packet loss rate, DoA is limited. But in case of time based aggregation, we have better DoA at the cost of delay and some packet loss. So lossy or lossless, count or time based aggregation is to be chosen based on the application type. Adaptive algorithm takes major role in getting the increased DoA based on the feedback.

\section{Conclusions}

The idea of the aggregation is to aggregate the data required close to the source or at intermediate nodes on the way to sink instead of sending all the sensor readings through the network. In this paper the idea of lossy and lossless aggregation has been proposed within a single algorithm. The proposed algorithm works for both lossy and lossless aggregation depending upon the requirement

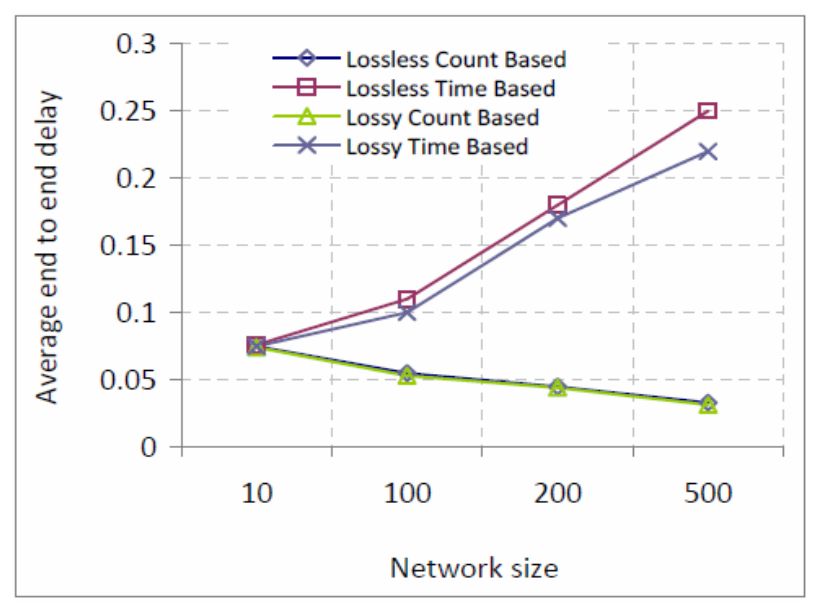

Figure 12. Average delay in aggregation for different network sizes. and makes the system adaptive to changes which can be adjusted with the load in the buffer and buffer management policy. The ultimate aim is to offer best QoS and significant savings in the energy and number of packets to be transmitted. The experiment has been carried out with Network simulator for large scale sensor network which advocates our proposed algorithm.

\section{References}

[1] B. Krishnamachari, D. Estrin, and S. Wicker, "Modelling data-centric routing in wireless sensor networks," in Proceedings of the IEEE INFOCOM, 2002.

[2] C. Intanagonwiwat, D. Estrin, R. Govindan, and J. Heidemann, "Impact of network density on data aggregation in wireless sensor networks," in Proceedings of the 22nd International Conference on Distributed Computing Systems (ICDCS'02), July 2002.

[3] V. Erramilli, I. Matta, and A. Bestavros , "On the interaction between data aggregation and topology control in wireless sensor networks," First Annual IEEE Communications Society Conference on Sensor and Ad Hoc Communications and Networks, IEEE SECON, pp. 557-565, 2004.

[4] A. Boulis, S. Ganeriwal, and M. B. Srivastava, "Aggregation in sensor networks: An energy-accuracy tradeoff," First IEEE International Workshop Sensor Network Protocols and Applications (SNPA'03), May 2003.

[5] Z. Ye, A. A. Abouzeid, and J. Ai, "Optimal policies for distributed data aggregation in wireless sensor networks," in Proceedings of 26th Annual IEEE Conference on Computer Communications (INFOCOM'07), Anchorage, Alaska, USA, May 6-12, 2007.

[6] T. He, B. Blum, J. Stankovic, and T. Abdelzaher, “AIDA: Adaptive application independent data aggregation in wireless sensor networks," ACM Transaction on Sensor Network, 2003.

[7] N. Shrivastava, C. Buragohain, D. Agrawal, and S. Suri, "Medians and beyond: New aggregation techniques for sensor networks," in Proceedings of the Second ACM Conference on Embedded Networked Sensor Systems (SenSys'04), August 2004.

[8] A. Deligiannakis, Y. Kotidis, and Roussopoulos, "Bandwidth-constrained queries in sensor networks," The VLDB Journal, Vol. 17, No. 3, pp. 443-467, 2008.

[9] TinyOS, http://www.tinyos.net/.

[10] K. Padmanabh and R. Roy, "Cost sensitive pushout policy and expelling polices with dynamic threshold for the buffer management in differentiated service switch for versatile traffic," IEEE International Conference on Networking, Mauritius, April 23-28, 2006.

[11] G. J. Fosdhini and B. Gopinath, "Sharing memory optimally," IEEE Transaction on Communication, Vol. 31, No. 3, pp. 352-360, March 1983.

[12] Network Simulator 2, http://www.isi.edu/nsnam/ns/. 